\title{
ESTUDO DE MOLUSCOS DO GÉNERO BIOMPHALARIA DE MINAS GERAIS, COM RELAÇÃO A ADAPTAÇÃO PARASITO HOSPEDEIRO E IMPORTÃNCIA NA EPIDEMIOLOGIA DA ESQUISTOSSOMOSE
}

\author{
Cecilia Pereira de souza
}

\section{R E S U M O}

Caramujos do gênero Biomphalaria das espécies B. tenagophila e B. straminea, descendentes de exemplares coletados em oito municípios mineiros, foram infectados com Schistosoma mansoni das cepas LE e SJ. As taxas de infecção experimental variaram de 0 a $28 \%$ para $\mathbf{B}$. tenagophila e de 0 a $21 \%$ para B. straminea. Esses resultados foram confrontados com os obtidos anteriormente por vários autores, mostrando que mais de $70 \%$ dentre 32 populações (doze de $\mathbf{B}$. te. nagophila e vinte de B. straminea) de Minas Gerais, foram suscetiveis experimentalmente a $\mathbf{S}$. mansoni. Os dados experimentais, aliados a relatos de encontro de B. tenagophila e B. straminea com infecção natural por $\mathbf{S}$. mansoni em quatro localidades a partir de 1982, parecem indicar que nessas regiōes existem condiçōes favoráveis de pré-adaptação ao parasitismo, a exemplo do que ocorreu no nordeste brasileiro e em São Paulo, pois, anteriormente moluscos dessas espécies não foram encontrados com infecção natural pelo trematódeo em Minas Gerais. Estes dados são importantes para o controle da disseminação da esquistossomose em áreas indenes, tendo em vista a vasta distribuição das duas espécies no Brasil.

UNITERMOS: Biomphalaria - adaptação parasito-hospedeiro. Esquistossomose Epidemiologia.

\section{N T R O D U G $\ddot{A} \mathbf{O}$}

A ampla distribuição geográfica de molus$\cos$ do gênero Biomphalaria, hospedeiros do Schistosoma mansoni, das espécies B. straminea e B. tenagophila, na América do Sul, a primeira na Venezuela, Guianas. Suriname, Brasil, Argentina, Paraguai e recentemente no Uruguai ${ }^{18}$ e a segunda, no Peru, Brasil, Argentina, Paraguai e Uruguai, demonstra a importância epidemiológica atual ou potencial desses pla. norbídeos frente ao fator adaptaçāo parasito hospedeiro.
Em Minas Gerais, região sudeste do Brasil, a distribuição geográfica das duas espécies é bastante ampla 23 mas elas não foram encontra. das com infecção natural por $\mathbf{S}$. mansoni até 1982. Nessa região a prevalência da esquistossomose em escolares foi de $9,6 \% 11$ e o molus. co hospedeiro é a B. glabrata, espécie predominante.

LUTZ em $1934{ }^{13}$ fez referência ao encontro de B. straminea (denominada Planorbis centimetralis naquela época), naturalmente infecta-

Trabalho realizado com auxílio do CNPq (Proc. 40.3659/82) e FINEP. Do Centro de Pesquisas "René Rachou" - Fundaçāo Oswaldo Cruz, M. S., C.P. 1743, 30000 Belo Horizonte-MG, Brasil.

Apresentado no I Colóquio Brasileiro sobre Esquistossomose, Fundaçāo Oswaldo Cruz, 1985. 
SOUZA, C. P. - Estudo de moluscos do gênero Biomphalaria de Minas Gerais, com relação a adaptaçãó parasito hospedei. ro e importância na epidemiologia da esquistossomose. Rev. Inst. Med. trop. São Paulo, 28:287-292, 1986.

da em Minas Gerais, fato näo confirmado durante cerca de 50 anos.

Os estudos de PARAENSE \& DESLANDES $19,20,21$, determinando as características sistemáticas das três espécies hospedeiras do S. mansoni no Brasil, deram uma valiosa contribuição para essas pesquisas, pois muitas vezes esses moluscos eram classificados como B. glabrata, por falta de maiores conhecimentos das diferenças morfologicas entre estas espé. cies.

A preocupação de pesquisadores com a possibilidade de moluscos B. straminea e B. tenagophila virem a se tornar hospedeiro do $\mathbf{S}$. mansoni em Minas Gerais é demonstrada por uma série de estudos sobre a suscetibilidade experimental dessas espécies $1,2,3,6,7,9,10,24,27,29$, $30,31,32$

No presente trabalho, em continuação a pesquisa iniciada anteriormente $29,30,31,32,33$ são apresentados dados sobre a suscetibilidade experimental de $\mathbf{B}$. tenagophila e $\mathbf{B}$. straminea de locais ainda não pesquisados, em Minas Gerais, e um arevisão desses estudos devido a sua importância epidemiológica.

\section{Material E MEtodos}

Foram utilizados caramujos B. tenagophila e B. straminea, criados em laboratório, descendentes de exemplares coletados, os primeiros, nos municipios de Betim Uberlânđia, Contagem (sítio e ranário) e Cabo Verde e $\mathbf{B}$. straminea, de Lagoa Santa, Mateus Leme, En. genheiro Caldas e Minas Novas. De cada população foram testados 200 exemplares, com diâmetro da concha variando de $4-7 \mathrm{~mm}, \mathbf{B}$. straminea e de 4-12 $\mathrm{mm}, \mathrm{B}$. tenagophila.

As cepas de S. mansoni usados foram: LE, de Belo Horizonte, mantida em laboratório há cerca de 20 anos e SJ, de São José dos Campos, SP, mantida em laboratório há 10 anos, mais adaptada a B. tenagophila, ambas man. tidas em B. glabrata.

Grupos de 50 caramujos de cada população foram expostos em massa a 50 ou $100 \mathrm{mi}-$ racídios por molusco. Para cada experimento foi feito um controle de infecção com B. glabrata do laboratório $(8.10 \mathrm{~mm})$, usando $20 \mathrm{mi}$ racídios/molusco.
A obtenção de miracídios e infecção de caramujos foram feitos segundo técnica descrita anteriormente ${ }^{28}$.

Decorridos 40 dias da infecção, os caramujos foram examinados individualmente em microscópio estereoscópico, após 30 minutos de exposição à luz e os exemplares positivos foram separados. Após mais 10 dias, os moluscos negativos foram examinados após esma gamento entre lâminas de vidro, procedimento usado também para examinar os exemplares mortos no decorrer da experiência. As taxas de infecção foram calculadas sobre o total de moluscos expostos sem levar em consideração o número de miracídios.

\section{RESULTADOS}

Os resultados obtidos anteriormente pelos diversos autores citados e os atuais, mostraram que nove dentre doze populaçōes de $\mathbf{B}$. tenagophila de Minas Gerais foram suscetíveis experimentalmente a $\mathbf{S}$. mansoni de cepa alóctone, São Paulo, o que está de acordo com observaçōes de PARAENSE \& CORREA ${ }^{22,25}$ e menos suscetiveis a cepas autoctones. As taxas de infecção experimental variaram de 0 a $95 \%$ (Tabela I) .

Quanto a B. straminea dezessete dentre vinte populações, foram suscetiveis experimentalmente a $\mathbf{S}$. mansoni de cepas autóctones ou alóctones. As taxas de infecção experimental foram mais baixas do que as de $\mathbf{B}$. tenagophiIa, não ultrapassando a $24 \%$ (Tabela II).

\section{DISGUSSÃO E CONCLUSÕES}

A evolução no sentido de adaptação para sito hospedeiro nas doenças parasitárias é uma realidade de grande importância epidemiológica.

Em esquistossomose existe vasta bibliogra fia sobre estudo e determinação da suscetibilidade de planorbideos de diferentes espécies à infecção pelo $S$. mansoni, o que demonstra a preocupação com o fator adaptação. Os fato. res que levaram a adaptação parasito hospe. deiro invertebrado, originando a esquistosso. mose nas Américas e no Brasil foram discutidos por MAGALHAES ${ }^{15}$, remontando à prová vel origem africana do $\mathbf{S}$. mansoni, introduzido 
SOUZA, C. P. - Estudo de moluscos do genero Biomphalaria de Minas Gerais, com relaçāo a adaptaçăo parasito hospedeiro e importância na epidemiologia da esquistossomose. Rev. Inst. Med. trop. São Paulo, 28:287.292, 1986.

T A B E I A I

Suscetibilidade experimental de B. tenagophila de Minas Gerais a Schistosoma mansoni

\begin{tabular}{|c|c|c|c|}
\hline \multirow{2}{*}{ Autor - (Ano) } & \multicolumn{2}{|c|}{ Procedência } & \multirow{2}{*}{$\begin{array}{l}\% \text { de } \\
\text { infecçẫo }\end{array}$} \\
\hline & Molusco & S. mansoni & \\
\hline Coelho (1962) & Itajubá * & MG & 0,0 \\
\hline \multirow[t]{2}{*}{ Paraense \& Correa (1978) } & Juiz de Fora & SP & 11,9 \\
\hline & Uba * & SP & 0,0 \\
\hline \multirow[t]{2}{*}{ Correa \& col. (1979) } & Belo Horizonte & MG & 0,0 \\
\hline & (Pampulha) & SP & 35,0 \\
\hline \multirow{2}{*}{\multicolumn{4}{|c|}{$\begin{array}{l}\text { Carvalho, Milward de } \\
\text { Andrade \& Souza (1979 a) Itajubá }\end{array}$}} \\
\hline & Itajubá & MG & 3,3 \\
\hline Carvalho \& Souza (1979 b) & Itajubá & SP & 95,0 \\
\hline Carvalho \& Souza (1980) & Sabará & MG & 1,25 \\
\hline \multirow[t]{8}{*}{ Souza \& col. (1983) } & Belo Horizonte & MG & 4,0 \\
\hline & (Horto) & SP & 22,0 \\
\hline & Sabará & MG (LE) & 0,0 \\
\hline & & $\mathrm{MG}$ & 2,0 \\
\hline & & $\mathrm{SP}$ & 6,0 \\
\hline & Nova Lima * & MG (LE) & 0,0 \\
\hline & & MG & 0,0 \\
\hline & & $\mathrm{SP}$ & 0.0 \\
\hline Souza & Betim & MG & 0,0 \\
\hline \multirow[t]{9}{*}{ (resultados atuais) } & & SP & 12,0 \\
\hline & Uberlândia & MG & 2,0 \\
\hline & (Ranário) & SP & 18.0 \\
\hline & Contagem & MG & 1,0 \\
\hline & (Ranário) & $\mathbf{S P}$ & 28,0 \\
\hline & Contagem & MG & 0,0 \\
\hline & (sítio) & SP & 24,0 \\
\hline & Cabo Verde & $\mathrm{MG}$ & 0,0 \\
\hline & & $\mathbf{S P}$ & 9,0 \\
\hline
\end{tabular}

\footnotetext{
- População resistente à infecção experimental pelo $\mathbf{S}$. mansoni
}

no continente pelos escravos e sua adaptação aos moluscos existentes na regiăo. Segundo esse Autor ${ }^{15}$, o grau de suscetibilidade do $\mathbf{S}$. mansoni ao molusco, estaria relacionado a variaçôes do genótipo das populaçōes do helminto e também a eventuais variaçōes intra-específicas do genótipo nas populações de molusco. Essas variações é que propiciariam condições de pré-adaptação ao parasitismo.

Estudos efetuados por RICHARDS ${ }^{26}$, sobre suscetibilidade de B. glabrata ao S. mansoni, indicam que esta é regulada por um complexo de vários fatores genéticos.

Os dados apresentados nesse trabalho re. velam que mais de $70 \%$ dentre 32 populaçōes de B. straminea (20) e de B. tenagophila (12), de Minas Gerais são suscetíveis experimentalmente a $\mathbf{S}$. mansoni e que algumas dessas populações, após período favorável de ajustamento parasito-hospedeiro, estão se transformando em hospedeiros intermediários do $\mathbf{S}$. mansoni,
T A B E I A II

Suscetibilidade experimental de $\mathbf{B}$. straminea de Minas Gerais à Schistosoma mansoni

\begin{tabular}{|c|c|c|c|}
\hline \multirow[b]{2}{*}{ Autor - (Ano) } & \multicolumn{2}{|c|}{ Procedência } & \multirow{2}{*}{$\begin{array}{l}\% \text { de } \\
\text { infecçāo }\end{array}$} \\
\hline & Molusco & S. mansoni & \\
\hline \multirow{3}{*}{$\begin{array}{l}\text { Fretas, Junqueira } \\
\text { \& Gerken (1972) }\end{array}$} & Lagoa Santa & $\mathbf{M G}$ & 0,60 \\
\hline & & MG & 1,04 \\
\hline & & SP & 0,90 \\
\hline $\begin{array}{l}\text { Gerken, Araujo \& } \\
\text { Freitas (1975) }\end{array}$ & Lagoa dos Mares & MG & 0,31 \\
\hline \multirow{3}{*}{$\begin{array}{l}\text { Souza, Rodrigues \& } \\
\text { Araujo (1981a) }\end{array}$} & Belo Horizonte & MG & 19,0 \\
\hline & & $\mathrm{MG}$ & $\mathbf{5 , 7}$ \\
\hline & & SP & 8,3 \\
\hline \multirow[t]{10}{*}{ Souza \& col. (1981b) } & Jequitibá & MG & 4,0 \\
\hline & & sp & 11,0 \\
\hline & Belo Oriente & MG & 0,0 \\
\hline & & SP & 3,0 \\
\hline & Juramento & MG & 5,0 \\
\hline & & $\cdots$ & 7,0 \\
\hline & Matozinhos & MG & 2,0 \\
\hline & (Moer "aro) & SP & 0,0 \\
\hline & Santana de & MG & 0,0 \\
\hline & Pirapama & SP & 7,0 \\
\hline \multirow{20}{*}{$\begin{array}{l}\text { Souza, Araujo \& } \\
\text { Azevedo (1983) }\end{array}$} & Matozinhos & MG & 2,0 \\
\hline & (Peri Perí) & SP & 15,0 \\
\hline & Betim & MG & 2,0 \\
\hline & & SP & 3,0 \\
\hline & Papagaios & MG & 1,0 \\
\hline & & SP & 11,0 \\
\hline & Sete Lagoas & $M G$ & 0,0 \\
\hline & & SP & 12,0 \\
\hline & Brumadinho * & MG & 0,0 \\
\hline & & $\mathbf{S P}$ & 0,0 \\
\hline & Chapada do & $M G$ & 0,0 \\
\hline & Norte & SP & 1,0 \\
\hline & Nova Lima & MG & 5,0 \\
\hline & & SP & 3,0 \\
\hline & Lontra & MG & 2,0 \\
\hline & & $\mathrm{SP}$ & 0,0 \\
\hline & & Al & 2,0 \\
\hline & Baldim & MG & 24,0 \\
\hline & & SP & 16,0 \\
\hline & & $\mathrm{AL}$ & 9,0 \\
\hline \multirow{8}{*}{$\begin{array}{l}\text { Souza (resultados } \\
\text { atuais) }\end{array}$} & Lagoa Santa & MG & 2,0 \\
\hline & (Represa Samambaia) & SP & 9,0 \\
\hline & Mateus Leme * & MG & 0,0 \\
\hline & & SP & 0,0 \\
\hline & Engenheiro Caldas & MG & 21,0 \\
\hline & & SP & 10,0 \\
\hline & Minas Novas • & MG & 0,0 \\
\hline & & $\mathbf{S P}$ & 0,0 \\
\hline
\end{tabular}

- População resistente à infecçãa experimental pelo $\mathbf{S}$ mansoni

a exemplo do que ocorreu no nordeste brasileiro e em São Paulo.

Os fatores que propiciaram esse ajusta mento, provavelmente foram as migrações internas de indivíduos portadores de esquistossomose ${ }^{16}$, as condições precárias de saneamento básico, em que vivem populaçōes de re 
SOUZA, C. P. - Estudo de moluscos do gênero Biomphalaria de Minas Gerais, com relaçāo a adaptação parasito hospedei. ro e importancia na epiđemiologia da esquistossomose. Rev. Inst. Med. trop. São Paulo, 28:287-292, 1986.

giōes peri-urbanas, cidades pequenas, vilas e povoados, aliados a alta densidade planorbídi. ca nos criadouros.

Em 1982, MELO, PEREIRA \& CORREIA 17 relataram o primeiro encontro de $\mathbf{B}$. tenagophila naturalmente infectada no município de Jaboticatubas ( $M G$ ), zona endêmica de esquis. tossomose, onde a B. glabrata era considerada - molusco hospedeiro responsável pela transmissão.

Em 1985, CARVALHO, SOUZA \& KATZ ${ }^{4}$ registraram o segundo encontro de $\mathbf{B}$. tenagophila naturalmente infectada com S. mansoni no sul de Minas Gerais, na cidade de Itajubá, região antes indene. Recentemente, CARVALHO \& col. ${ }^{5}$ encontraram B. temagophila natural. mente infectada no lago da Pampulha, Belo Horizonte (MG), local pesquisado ao longo de vários anos, com registro apenas de B. glabra. ta albergando S. mansoni.

Quanto à B. straminea, cincoenta anos após o relato de LUTZ ${ }^{13}$, DIAS PINTO \& $\mathrm{col}^{8} .^{8}$ registraram o segundo encontro desse planorbideo com infecção natural por $\mathbf{S}$. mansoni em Minas Gerais, na Represa de Samambaia, divisa dos municipios de Lagoa Santa e Pedro Leopoldo.

Estudos realizados por vários autores sobre a população de B. tenagophila de Itajubá, sul de Minas Gerais, mostram o que pode estar ocorrendo em regiöes onde existem moluscos dessas espécies.

Após o registro da existência de B. tenagophila ( = Australorbis nigricans) em Itajubá em $1955^{20}$, COELHO (1962) 6 tentou sem sucesso infectar experimentalmente esse planorbídeo com S. mansoni de Belo Horizonte. Decorridos dezessete anos, CARVALHO, MIL. WARD DE ANDRADE \& SOUZA' e CARVALHO \& SOUZA ${ }^{2}$ tentaram novamente infectar esses moluscos com S. mansoni de Belo Horizonte e de São José dos Campos (SP). Os resultados foram positivos com ambas as cepas e a taxa de infecção foi mais elevada com miracídios de Săo José dos Campos, mostrando maior adaptação à $\mathbf{B}$. tenagophila daquela região vizinha.

Em levantamento malacológico realizado em Itajubá, em 1983 '2, foram capturados 1995 exemplares de B. tenagophila e 91 de B. peregrina mas nenhum albergava cercárias ou esporocistos de S. mansoni. Um novo levantamento malacológico foi realizado em 19854 , sendo capturados 1501 exemplares de B. tenagophila dos quais $2(0,14 \%)$ eliminavam cercárias de $\mathbf{S}$. mansoni. A ocorrência de casos autóctones de esquistossomose na região aumentou de três, relatados em $1983^{12}$, para mais de duzentos em 1985, segundo relatos de técnicos que trabalham no Centro de Saúde local. Como, no último levantamento, só foi encontrada B. tenagophila, essa espécie provavelmente é a responsável pelo novo foco, ficando a manutenção do mesmo garantida pelo doente autóctone, portador de cepa de $\mathbf{S}$. mansoni préadaptada ao molusco local ${ }^{14}$.

Espécies de moluscos do mesmo gênero, como B. peregrina 12 e B. schrammi ${ }^{33}$, não foram encontradas com infecção natural por $\mathbf{S}$. mansoni em Minas Gerais.

Mediante os registros efetuados por dife. rentes pesquisadores conclui-se que a adaptação parasito-hospedeiro ocorrida nas regiões estudadas é de grande importancia na epidemiologia da esquistossomose. Tendo em vista a vasta distribuição geográfica das duas espécies no Brasil e também na América do Sul e o fator migrações humanas, ressalta-se a importância desses relatos para o controle da disseminação da esquistossomose em areas indenes.

\section{SUMMARY}

Study of molluses of the genus Biomphalaria from State of Minas Gerais in regard with host parasite adaptation and importance in epidemiology of Schistosomiasis.

Biomphalaria tenagophila and B. straminea snails bred from snails collected at 8 counties of Minas Gerais, Brazil, were infected with Schistosoma mansoni miracidia of the strains $L E$ and $S J$. The percent experimental infection range was $0.28 \%$ for $B$. tenagophila and $0-21 \%$ for B. straminea. These results were compared with the ones by other authors showing that more than $70 \%$ of 32 populations from Minas Gerais (12 populations of $\mathbf{B}$. tena gophila and 20 of B. straminea) were suscep- 
SOUzA, C. P. - Estudo de moluscos do gênero Biomphalaria de Minas Gerais. com relaçāo a adaptaçāo parasito hospedelro e importância na epidemiologia da esquistossomose. Rev. Inst. Med. trop. São Paulo, 28:287-292, 1986.

tible to experimental infection with S. mansoni. These experimental data together with reports claiming the finding of naturally $\mathbf{S}$. mansomi infected B. tenogophila and B. straminea at four localities since 1982 seem to indicate the existance of favourable conditions to the pre-adaptation to parasitism, as no infected snails had been found in these regions before. This adaptation has been described to occur in São Paulo and in brazilian northeastern region. These data are important to the control of the dissemination of schistosomiass to undamaged areas, as these two species of Biomphalaria are widely distributed in Brazil.

\section{AGRADECIMENTOS}

A Dra. Neusa Araújo pela colaboração na parte expetimental.

\section{REFERENCIAS BIBLIOGRAFICAS}

1. CARVALHO, O. S.; MILWARD DE ANDRADE, R. \& SOUZA, C. P. - Susceptibilidade de Biomphalaria tenagophila (d’Orbigny, 1835), de Itajubá (MG) à infecção pela cepa "LE" de Schistosoma mansoni Sambon, 1907, de Belo Horizonte, MG (Brasil). Rev. Saúde públ. (S. Paulo), 13: 20-25, 1979.

2. CaRvalHo, O. S. \& SOUZA, C. P. - Comportamento de Biomphalaria tenagophila (d'Orbigny, 1835) de Itajubá (MG, Brasil), exposta à cepa "SJ" de Schistosoma mansoni Sambon, 1907. In: CONGRESO DA SOCIEDADE BRASILEIRA DE PARASITOLOGIA, 4.․ Campinas, 1979. Resumos. Campinas, Universidade Esta dual de Campinas. 1979. p. 105.

3. CARVALHO, O. S. \& SOUZA, C. P. - Suscetibilidade de Biomphalaria tenagophila (d'Orbigny, 1835) ori. ginárias de Ravena, Municipio de Sabará, MG (Brasil), a cepa "LE" de Schistosoma mansoni. In: CONGRESSO BRASILEIRO DE PARASITOLOGIA, 5.․ Rio de Janeiro 1980. Resumos. Rio de Janeiro, FIOCRUZ, 1980. p. 152

*. Carvalho, O. S.; SOUZa, C. P. \& KaTz, N. Primeiro encontro de Biomphalaria tenagophila (d'Or: bigny, 1835) naturalmente infectacia com Schistosoma mansoni, em Itajubá, Sul do Estado de Minas Gerais, Brasil. Rev. Saúde públ. (S. Paulo), 19: 88-91. 1985.

5 CaRValHo, O. S; GUIMARAES, C. T'.; MASSARA; C. L. \& BONESIO, J. E. R. - Situação atual da esquistossomose mansoni no lago da Pampulha, Belo Horizonte, MG, Brasil. Rev. Saúde públ. (S. Paulo), 39: $270-277,1985$

6. COELHO. M. V. - Suscetibilidade de Australorbis tenagophilus à infecçăo por Schistosoma mansoni. Rev. Inst. Med. trop. S. Paulo, 5: 289.295, 1962.

7. CORREA, M. C. R.: COELHO, P. M. Z. \& FREITAS, J, R. - Susceptibilidade de linhagens de Eiomphalaria tenagophila e Bomphalaria glabrata a duas cepas de Schistosoma mansoni (LE - Belo Horizonte e SJ São Jose dos Campos). Rev. Inst. Med. trop. S. Paulo, 21: $72-76,1979$.

8. DIAS PINTO, A. M. S.; BIZOTTO PINTO, C. M; FERREIRA, H. L. M.; ASSIS, L. . S.; ROLIA, M. E \& JUNQUErRA, M. v. - Presença de Biomphalaria straminea naturalmente infestada pelo Schistosoma mansoni na Represa Samambaia, divisa dos municípios de Lagoa Santa e Pedro Leopoldo, MG, março de 1981. Ciêne. e Cult. 36 (Supl.): 893, 1984.

9. FREITAS, J. R.; JUNQUEIRA, D. V. \& GERKEN, S. E. - Habitats primitivos de hospedeiros do S. mansonj na regiāo de Lagoa Santa, MG. Ciênc. e Cult., 24: 377,1972

10. GERKEN, S. E.; ARAUJO, M. P. T. \& FREITAS, J. R. - Suscetibilidade de Biomphalaria straminea da re. giän de Lagoa Santa (MG) ao Schistosoma mansoni. Rev. Inst. Med. trop. S. Paulo, 17: 338.343, 1975.

11. KATZ, N.: MOT'A, E.; OLIVEIRA, V. B. \& CAR. VALHO, E. F. - Prevalência da esquistossomose em escolares no Estado de Minas Gerais. In: CONGRESSO DA SOCIEDADE BRASILEIRA DE MEDICINA TRO. PICAL, 14. e CONGRESSO DA SOCIEDADE BRASILEIRA DE PARASITOLOGIA, 3.', Joāo Pessoa, 1978. Resumos dos Temas Livres. Joảo Pessoa, Editora Universitéria, 1978, jo. 102.

12. KATZ, N. \& CARVALHO, O. S. - Introduçăo tecente de esquistossomose mansoni no sul do estado de Minas Gerais, Brasil. Mem. Inst. Osw. Cruz, 78: 281-284, 1983.

13. LUTZ, A. - Transmission du Schistosoma mansoni dans l'Etat de Minas Gerais (Brésil) par le Planorbis centimetralis. C. R. Soc. Biol. (Paris), 116: J149-1150, 1934.

14. Magalhäes, L A.: CAMARGO, L. A. P.: MUNiZ, J. R. O. \& ANDRADE, D. - Um novo foco de esquis. tossomose mansoni na cidade de Campinas (Estado de Sâo Paulo, Brasij). Rev. Inst. Med. trop. S. Paulo, 9; 378-380, 1967.

15. MagalhaES, L. A. - Estudo do comportanento de cepa de S. mansoni de Brasilia. Hospital (Rio de J.). 11: $273.282,1970$.

16. MARQUES, A. C. - Migraçöes internas e as grandes endemias. Rev, bras. Malar., 31: 137.158, 1979

17. MELO, A. L.; PEREIRA, L. H. \& CORREA, M. C. R - Sobre o encontro de Biomphalaria tenagophila natu ralmente infectada com Schistosoma mansoni no mu nicípio de Jaboticatubas, Minas Gerais. In: CONGRES SO BRASILEIRO DE PARASITOKOGIA, 8.0, j982. Re sumos. p. 63.

18. OLAZARRI, O, - Bjomphalaria stramimea (Dunker 1848) (Mollusca: Gastropoda) on la cuenca del rio Uruguay Hist. nat.. 4: 285-288, 1984. 
SOUZA, C. P. - Estudo de moluscos do gênero Biomphalaria de Minas Gerais, com relação a adaptaçāo parasito hospedeiro e importância na epidemiologia da esquistossomose. Rev. Inst. Med. trop. Sãa Paulo, 28:287-292, 1986.

19. PARAENSE W. L. \& DESLANDES, $N$, - Observations on the morphology of Australorbis glabratus. Mem. Inst: Osw, Cruz, 43: 87.103, 1955a.

20. PARAENSE, W. L. \& DESLANDES, N. - Observations on the morphology of Australorbis nigricans. Mem. Inst. Osw. Cruz, 53; 121-134, 1955b.

21. PAREENSE, W. L. \& DESLANDES, N. - Studies on "Australorbis centimetralis". II: Bioespecific characterization. III Generic status. Rev. bras. Biol., 15: 341-348, $1955 \mathrm{c}$.

22. PARAENSE, W, L. \& CORREA, L. R. - Sobre a ocorrência de duas raças Biológicas de Schistosoma mansoni no Brasil: Resumos XV Reuniáro Anual Soc. Brasil. Progresso Cienc. Campinas, SP, $1963 \mathrm{a}$.

23. PARAENSE, W. L. - Fauna planorbídica do Brasil. In: LACAZ, C. da S.; BARUZZI, R. G. \& SIQUEIRA

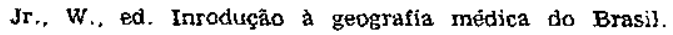
Sāo Paulo, Edgara Blucher; Editora da Universidade de São Paulo, 1972. Cap. 10, p. 213-239.

24. PARAENSE, W. L. \& CORREA, L. R. - Differential susceptibility of Biomphalaria tenagophila populations to infection with a strain of Schistosoma mansoni. J. Parasit., 64: 822-826, 1978.

25. PARAENSE, W. L. \& CORREA, L. R. - Observations on two biological races of Schistosoma mansoni. Mem. Inst. Osw. Cruz, 76: 287-291, 1981.

26. RICHARDS, C. $\mathbf{S}$. - Influence of snail age on genetic variations in susceptibility of Biomphalaria glabrata for infection with Schistosoma mansoni. Malacologia. 25: 493.502, 984 .

27. SANTOS, M. B. L.; FRETTAS, J. R.; CORREA, M. C. R. \& COELHO, P. M. Z. - Suscetibilidade ao Schistosoma mansani de hibridos de Biomphatarja tenagophila do
Taim, RS, Cabo Frio, RJ e Belo Horizonte, MG. Rev. Inst. Med. trop. S. Paulo, 21: 281-286, 1979.

28. SOUZA, C. P.; DIAS, E. P.: AZEVEDO, M. L. L. \& PAULINI, E. - Observaçōes sobre alguns fatores quo influem na manutenção do Schistosoma mansonI em laboratório. Rev. bras. Pesq. méu. biol., 12: 411-419. 1979 .

29. SOUZA, C. P.; RODRIGUES, M. S. \& ARAUJO, N. - Suscetibilidade de Biomphalaria straminea (Dunker. 1848) de Belo Horizonte (MG) á infecçāo por cepas de Schistosoma mansoni. Rev. Inst. Med. trop. S. Paulo, 23: 188-193, 1981a.

30. SOUZA, C. P.; RODRIGUES, M. S.; AZEVEDO, $M$, L. L. \& ARAUJO, N. - Suscetibiliaade de populaçōes de Biomphalaria straminea (Dunker, 1848) de Minas Gerais à infecçāo por Schjstosoma mansoni. Rev. Inst. Med. trop. S. Paulo, 23: 212-216, 1981b.

31. SOUZA, C. P.; ARAUJO, N. \& AZEVEDO, M. L. L. Estudio da potencialidade de populaçōes de Biompha. laria straminea do Estado de Minas Gerais, como hospedeiras do Schistosoma mansont, Mem. Inst. Osw. Cruz, 28: 251.256, 1983a.

32. SOUZA, C. P.; ARAUJO, N.; MADEIRA, N. G. \& CAR. VALHO, O. S. — Suscetibitidade de Blomphalaria tenagophila de Belo Horizonte $\theta$ adjacências à infecção com três cepas de Schistosoma mansoni. Rev. Inst. Med. trop. S. Paulo. 25: 168.172, $1983 \mathrm{~b}$.

33. SOUZA, C. P.; GUMMARAES, C. T.; ARAUJO, N. \& SILVA, C. R. T. - Resistēncia de Biomphalaria schram. mi de ATcos, Minas Gerais-Brasil à infecç̃o com duas cepas do Schístosoma mansoni. Mem. Inst. Osw. Cruz. 80: $51-53,1985$.

Recebido para publicação em 29/10/1985 